SEISMIC LOVE WAVES

POVERKHNOSTNYE VOLNY LYAVA

ПОВЕРХНОСТНЫЕ ВОЛНЫ ЛЯВА 


\title{
SEISMIC LOVE WAVES
}

\author{
Z. S. Andrianova V. I. Keilis-Borok \\ Institute of Chemical Physics Institute of Physics of the Earth \\ A. L. Levshin M. G. Neigauz \\ Institute of Physics of the Earth Institute of Chemical Physics \\ Academy of Sciences of the USSR
}

Translated from Russian by

F. M. C. Goodspeed

Department of Mathematics

Laval University

Quebec, Canada

(

CONSULTANTS BUREAU • NEW YORK 1967 


\title{
About the Authors:
}

Zoya Sidorovna Andrianova was born in 1938. She was graduated from Moscow University in 1960 and is now at the Institute of Chemical Physics of the Academy of Sciences of the USSR. Andrianova is the author of several articles on the numerical solution of differential equations.

Vladimir Isaakovich Keilis-Borok was born in 1921 and was graduated from the Institute of Geological Exploration in 1943. He received his doctorate in physical mathematics in 1953. Director of the interpretation-methods section of the Institute of Physics of the Earth of the Academy of Sciences of the USSR, he is the author of the monograph "Interference Surface Waves." His principal work concerns the problems of elastic-wave theory, the machine interpretation of seismic observations, and the detection of nuclear explosions.

Anatolii L'vovich Levshin was born in 1932 and was graduated from Moscow University in 1954. He is now at the Institute of Physics of the Earth of the Academy of Sciences of the USSR studying the theory and interpretation of surface waves in engineering seismic exploration and seismology.

Militsa Genrikhovna Neigauz was born in 1929, and was graduated from Moscow University. She is now at the Institute of Chemical Physics of the Academy of Sciences of the USSR and is associated with the mathematical school of I. M. Gel'fand. She is the author of several articles on the spectral theory of operators, optimum control, and stability theory.

The Russian text, originally published by Nauka Press in Moscow in 1965 for the Institute of Physics of the Earth and the Institute of Chemical Physics of the Academy of Sciences of the USSR, has been extensively corrected and expanded by the authors for the English edition.

3. С. Андрианова, В. И. Кейлис-Борок, А. Л. Левщин, М. Г. Нейгауз

\section{Поверхностные волны Лява}

Library of Congress Catalog Card No. 66-19935

\author{
ISBN 978-1-4684-8606-3 ISBN 978-1-4684-8604-9 (eBook) \\ DOI 10.1007/978-1-4684-8604-9

\section{(1) 1967 Consultants Bureau}

Softcover reprint of the hardcover 1st edition 1967

A Division of Plenum Publishing Corporation

227 West 17 Street, New York, N. Y. 10011

All rights reserved

No part of this publication may be reproduced in any form without written permission from the publisher 


\section{FOREWORD TO THE ENGLISH EDITION}

Despite the fact that there have been a great many studies of Love waves, the present book is not a summary of previous work; it contains new practical results and is completely dedicated to questions of method. This requires an explanation of why we should consider methods of investigation which are already very effective. The explanation is related to the development of the problem.

The electronic computer and new recording apparatus have recently and suddenly revealed great new possibilities in the investigation of surface waves. The new potentialities naturally first developed in conjunction with a method which, in reference to petroleum resources in the USA, is called wildcatting. Here investigators try to obtain specific results without any interest in the optimum nature of the method, in the uniqueness of the results, in the investigation of allied problems, etc.

We have a high regard for the useful results obtained and the attempts made during this period. It is clear, however, that we have arrived at a stage in which better planned and more complex investigations must be carried out. At this time we need a summary of the general fundamental aspects of the problem. We refer to the general theoretical properties of Love waves, to methods for calculating the parameters of Love waves, to the relations between the properties of Love waves and various features of the medium and the source, to the accuracy of the observations needed for the determination of various details of profiles of the crust or mantle, to the uniqueness of the results of interpretation, etc.

These general problems are considered in the present book. A short summary of the contents is given below in the introduction. The text has been completely revised and an appendix added for the present edition; the main additions to the original text are devoted to a consideration of the influence of sphericity and absorption and an analysis of theoretical seismology. The appendix was written with the participation of $\mathrm{E}$. V. Vil'kovich and N. P. Grudeva.

We believe that this book will be of interest to English-speaking readers who are interested in Love waves, even though numerous articles on this subject have been published in the English technical literature.

The Authors 


\section{PREFACE}

Among seismic waves generated by widely diiferent types of sources, the waves that are the most interesting and of the longest duration are usually surface waves (which are also called normal waves, interference and channel waves, natural vibrations of layers, etc.). These waves are distinguished by their dispersion and resonance, and are used in many investigations - in the determination of the structure of the medium (the existence of surface layers and waveguides), in the determination of the coordinates and properties of the source (in particular its energy and mechanism), in the identification of subterranean explosions, in the mapping of microseisms, in the tracing of storms, etc. Outlines of the wide literature concerning surface waves can be found in $[2,9,40]$.

Surface waves can be classed as Rayleigh or Love waves, depending on their velocity and polarization: the displacements of particles in Rayleigh waves are parallel, and the displacements in Love waves perpendicular, to the vertical plane containing the direction of propagation (some variation from this polarization is possible close to the source or in the presence of horizontal inhomogeneities in the medium).

The present work is devoted to the calculation and investigation of Love waves in a vertically layered medium. The method used is based on the spectral theory of linear differential operators. ${ }^{*}$ This method is much more effective than previously applied methods based on the approximation of the medium by a set of homogeneous layers.

Chapter I describes the theory of Love waves. In it we make the following assumptions: The medium is a perfectly elastic, isotropic half-space, the parameters of which are piecewise continuous functions of the depth, independent of the horizontal components. The contact between particles on the planes of discontinuity is assumed to be perfect. Beginning at a certain depth the parameters of the medium become constant and the volume-wave velocity reaches its maximum. In the source, the volume forces are distributed symmetrically relative to a vertical axis. The dependence of the force in the source on the time and on the horizontal coordinates is assumed to be sufficiently smooth (the conditions imposed on the source can be somewhat relaxed but this is not physically essential).

In Section 1 we give the original formulas. By using the ordinary method of separation of variables and some extra calculations, we express the nonstationary displacements in Love waves in a three-dimensional medium (and in particular their asymptotic representation at large horizontal distances from the source) in terms of the characteristic functions of a Sturm-Liouville operator.

In Section 2 we consider a method for the calculation and investigation of Love waves. This method yields not only the dispersion, but also the intensity - in practice for any mode and any velocity and density profile in a wide range of frequencies. A program is written for using this method with an electronic digital computer.

*This theory has previously been used only in the qualitative investigation of the dispersion of Love waves in an inhomogeneous layer on an absolutely rigid half-space [43]. 
In Section 3 we consider the fundamental theoretical properties of Love waves: * their dispersion; the dependence of their intensity on the vibration frequency, the source depth, and the mode number; the nature of nonstationary vibrations and methods of constructing theoretical seismograms; the laws of similarity and the reciprocity principle. We describe in detail the specific properties of vibrations in the presence of waveguides. In conclusion we give the basic information needed for the application of the program that has been built up.

Chapters II and III contain a discussion on the possibility of using Love waves for the investigation of the structure of the crust and the upper mantle; it is explained what observations are necessary for the determination of certain profile elements (waveguides, zones of higher gradient, boundaries between layers), and what details of a profile can be determined from given observations (one or several of the layer modes). The two chapters are similarly planned.

Sections 4 and 7 contain descriptions of frequency characteristics; from them we can determine the range of periods and focal depths for which the first three or four modes are sufficiently intense. The resulting frequency characteristics are used in deciphering the wave picture, in obtaining source depths, and in obtaining more accurate earthquake magnitudes; they can also be used in the analysis of microseisms, the investigation of resonance properties of layers, etc.

Calculations show a very slight decrease in intensity with increasing mode number; in some examples there is almost no variation in the intensity of the first forty modes. We have not yet succeeded in establishing whether real records are actually formed by the superposition of such a great number of modes. We therefore confine ourselves to the consideration of those time intervals and vibration periods for which only the first 3-4 modes are recorded.

In Sections 5 and 8 we compare the phase- and group-velocity dispersion of these modes for various velocity profiles. We explain which sections of the dispersion curves are needed for the determination of those elements of a profile which are of direct interest in seismology. The basic lack of definiteness in several common methods of interpretation is demonstrated. Thus from the first (fundamental) mode we can determine for the earth's crust only a certain approximately linear combination of thickness and mean velocity, and this only when the thicknesses and the mean velocities of the layers covering the strictly crystalline crust are known; for the upper mantle we can determine only the mean velocity over the large depth intervals (of the order of hundreds of kilometers), while waveguides can remain undetected if their parameters are close to those which can be received at present.

Methods are described for overcoming this lack of definiteness and for determining the basic details of a profile. These methods reduce to the simultaneous employment of several modes (appropriate to the frequency intervals employed) or of body and surface waves. However, for any conceivable system of observations, interpretation must be made in terms of a set of profiles, all of which may satisfy the data, rather than a single, unique profile.

The best possible prospect of determining waveguides is clearly in the use of specific vibrations related to a secondary group-velocity minimum of the third mode.

In Section 8 we compare some calculated results with experimental observations. An example is given of the simultaneous interpretation of surface and body waves; a group of profiles is found compatible with the observed dispersion of the first mode of Love waves in the travel time of the $S$ Wave.

In Sections 6 and 9 we clarify the conditions for obtaining data required for the solution of the problems considered in Sections 5 and 8. Theoretical seismograms are obtained for nonstationary Love waves. Criteria are given for the separation of the different modes.

Chapter IV contains a discussion of the relation between the nature of the source and the observations of Love waves. The possibility is considered of estimating the focal depth (when the focus is in the crust) from

*Readers who are not interested in the purely mathematical side of the problem can begin with Section 3 , which starts with the basic definitions. 
the spectrum of the fundamental mode (Section 10); corrections of the magnitude for focal depth are considered in Section 11; the relation between the azimuthal distribution of intensity and the mechanism of an earthquake is discussed in Section 12.

This summary of the contents of the book has left the authors with a feeling of some dissatisfaction. If we were rewriting the book we would probably have the models of the medium in Sections 5 and 8 vary automatically (i.e., by using the appropriate program) as was done in the solution of the inverse problem ([28, 32], Paragraph 2, Section 8 of this book), and would investigate the derivatives of the phase and group velocity with respect to the profile parameters. We would also investigate the quantitative correlation between profiles and group-velocity curves for higher modes.

We decided, however, not to pospone the publication of the book for consideration of the above questions in the hope that it can, in its present form, at least partially show the great possibilities inherent in the use of Love waves for seismology.

In the preparation of the mathematical part of the book we had the valuable advice of Professor V. B. Lidskii, Professor A. Ya. Povzner, and Doctor of Mathematical and Physical Sciences I. I. Pyatetsk-Shapiro. Formulation of the interpretational problems was greatly helped by the advice of B. Ya. Gel'chinskii. Members of the Leningrad Division of the Mathematical Institute, Academy of Sciences USSR I. Ya. Azbel' and T. B. Yanovskii, and of the Institute of Physics of the Earth, Academy of Sciences USSR V. P. Valyus and N. P. Grudev allowed us to publish a series of programs and calculations. Valuable suggestions concerning the contents of Chapter I were made by A. A. Gvozdev and Z. A. Yanson.

The authors thank the above specialists, without whose help the work could not have been completed.

We must also express our gratitude to our fellow workers in the laboratories of the Institute of Physics of the Earth, Academy of Sciences USSR, R. V. Abramova, E. G. Dirdovska, M. G. Koldaeva, and L. N. Pozharska, who performed all the technical work in the analysis of calculations and the preparation of the book. 


\section{CONTENTS}

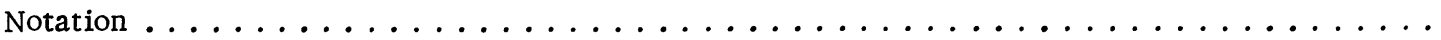

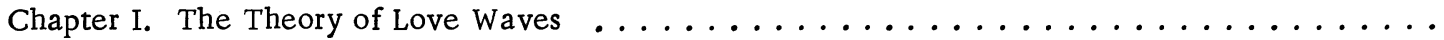



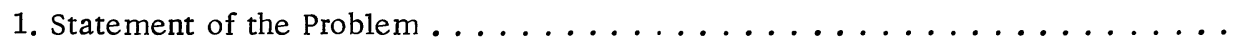

2. Expressions for Displacements in Terms of the Solution of Two Plane Problems ..

3. Expressions for Displacements in Terms of Characteristic Functions of the



4. An Asymptotic Representation of the Displacement Spectrum . . . . . . . . .

5. Modes of Surface Waves and their Phase Velocities ..............

6. An Asymptotic Formula for Nonstationary Displacements . . . . . . . . . . .

$\S 2$. Methods of Calculating the Parameters of Love Waves . . . . . . . . . .

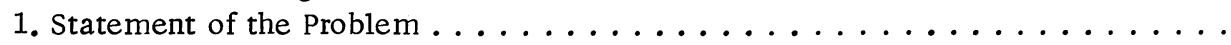

2. The Solution of the Problem for Fixed Frequencies . . . . . . . . . . .

3. A Method of Solving the Initial-Value Problem for Eq. (2.3) . . . . . . . . .

4. The Calculation of Characteristic Values .................

$\S 3$. The Basic Properties of Love Waves . . . . . . . . . . . . . . . .

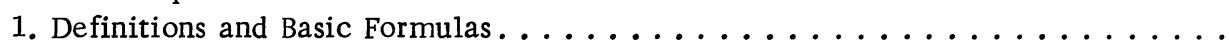

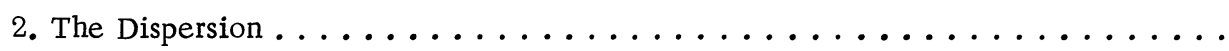

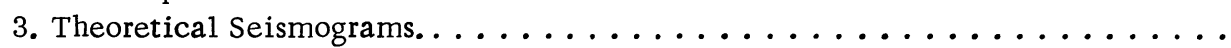



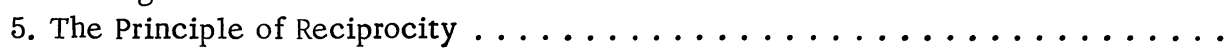

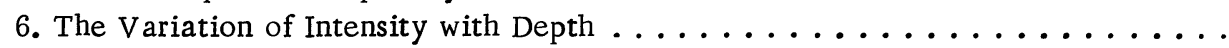

7. The Dependence of the Intensity on the Frequency and the Mode Number .....

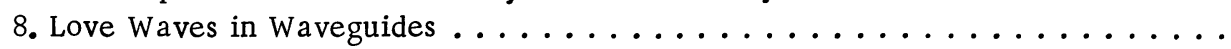

9. A Program for the Calculation of Dispersion and Intensity . . . . . . . . .

Chapter II. Love Waves in the Earth's Crust . . . . . . . . . . . . . . . . .

$\S 4$. Frequency Characteristics of the Medium ..................

1. The Fundamental Mode; A Crust without a Surface Layer . . . . . . . . . . .

2. The Fundamental Mode; A Crust with a Surface Layer . . . . . . . . . . .

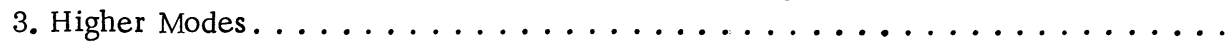

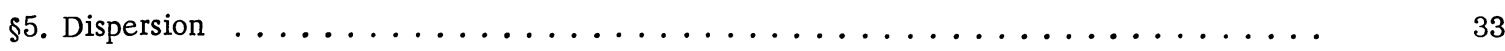

1. The Fundamental Mode; Crusts without Surface Layers . . . . . . . . . . . . . . . . . . . . . . 34

2. The Fundamental Mode; Crusts with Surface Layers . . . . . . . . . . . . . . . . . . . . 37

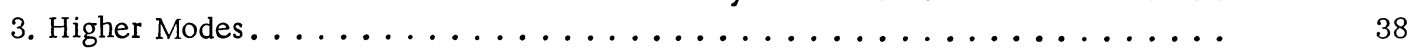

$\S 6$. The Theoretical wave Picture . . . . . . . . . . . . . . . . . . . . . 41

Chapter III. Love Waves in the Upper Mantle . . . . . . . . . . . . . . . . . . . 47 


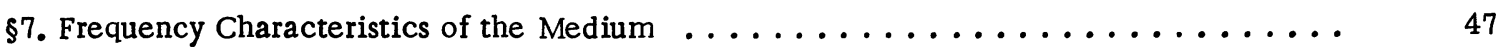

1. The Fundamental Mode ........................... 47

2. Higher Modes................................. 48

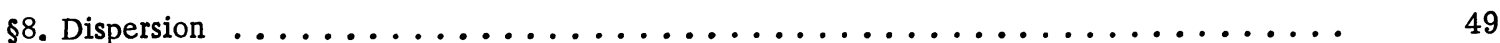

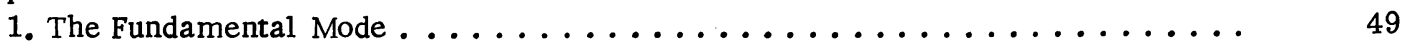

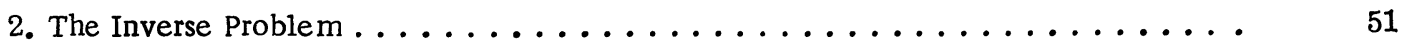

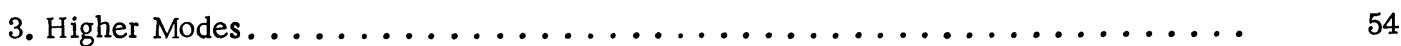

$\S 9$. The Theoretical Wave Picture............................ 61

Chapter IV. Love Waves and the Parameters of the Source . . . . . . . . . . . . . . . . . . . . . . . . . .

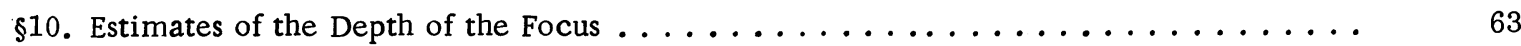

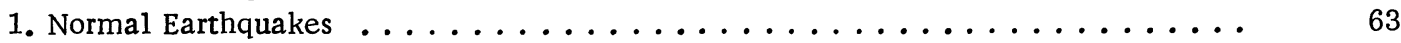

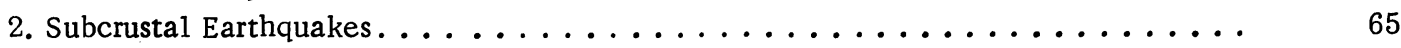

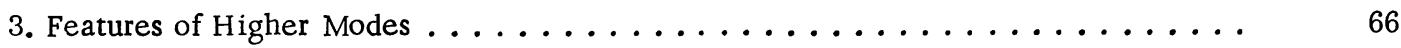

§11. The Determination of the Magnitude $M$ with the Depth of the Focus taken into Account. 67

1. The Depth of Penetration of Waves Used in the Determination of Magnitude. . . . 67

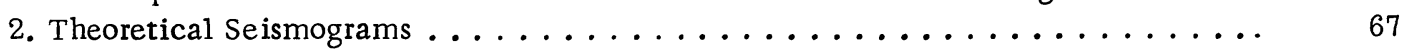

$\S 12$. The Azimuthal Intensity Distribution of Love Waves. . . . . . . . . . . . . . 69

Appendix. The Influence of Sphericity, Variation of the Parameters, and Absorption . . . . . . . . 75

$\S 13$. The Influence of the Sphericity of the Earth . . . . . . . . . . . . . . . . . . . . . . . . . . . .

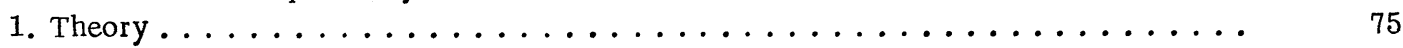

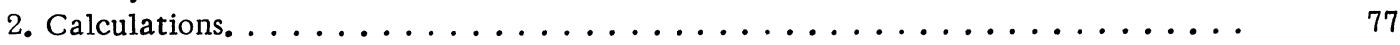

$\S 14$. Derivatives of the Phase and Group Velocities with Respect to the Parameters

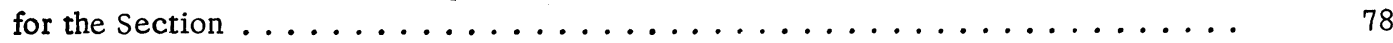

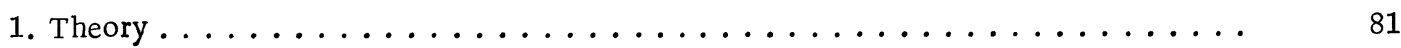

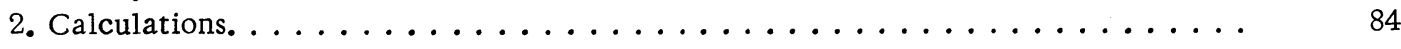

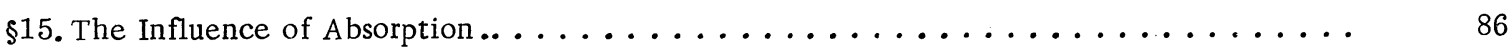

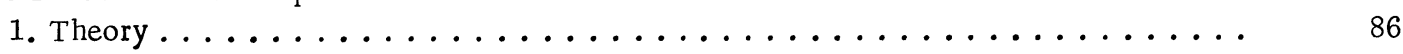

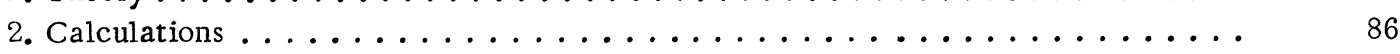

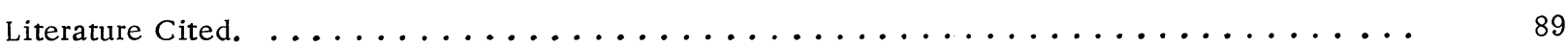




\section{NOTATION}

a - longitudinal-wave velocity

$b$ - transverse-wave velocity

$b_{0}$ - transverse - wave velocity at the surface of a crystalline earth's crust

$\mathrm{b}_{\mathrm{M}}$ - transverse-wave velocity in the mantle directly under the Mohorovicic discontinuity

$\mathrm{b}(\tilde{z})-$ minimum velocity of transverse waves in the mantle

$\overline{\mathrm{b}}$ - mean transverse-wave velocity in the crust

$\mathrm{C}_{\mathrm{k}}$ - group velocity of the $\mathrm{k}$-th mode (1.62)*

$c_{k}^{L}$ - coefficients of the expansion of the displacement spectra of Love waves in characteristic functions (1.35)

$\mathrm{C}_{\mathrm{k} l}^{\mathrm{R}}$ - coefficients of the expansion of the displacements in Rayleigh waves in characteristic functions (1.42)

E - dipole parameter (3.3)

$\mathrm{F}_{l}$ - component of volume forces at the source along the $l: l=\mathrm{T}$ is the horizontal component, $l=\mathrm{z}$ is the vertical component (1.11)

$\widetilde{\mathrm{F}}_{l}$ - space-time spectrum (the double Fourier transform) of $\mathrm{F}_{l}(1.11)$

$G$ - dipole parameters (3.3)

$\mathrm{H}$ - thickness of the earth's crust

$\mathrm{h}-$ focal depth (1.56)

$\mathrm{J}_{\mathrm{L}}$ - norming factor for the calculation of Love -wave intensity (1.36)

$\mathrm{J}_{\mathrm{R}}$ - norming factor for the calculation of Rayleigh-wave intensity (1.43)

$\mathrm{k}$ - mode number (characteristic value), $\mathrm{k}=1,2, \ldots$

$k_{L}(p)$ - mode number of Love wave characteristic values of the operator $(1.21)-(1.22)$ existing for a given $\mathrm{p}(1.34)$

$k R(\xi)$ - mode number of Rayleigh waves [characteristic values of the operator (1.16)-(1.20)] existing for given $\xi(1.40)$

$\mathrm{L}$ - proportionality coefficient in similarity laws (3.10)

$M$ - magnitude with respect to surface waves (11.1)

$\mathrm{m}$ - three-dimensional frequency corresponding to the variable $\mathrm{x}(1.9)$

$\mathrm{n}$ - three-dimensional frequency corresponding to the variable $y(1.9)$

$p$ - time frequency corresponding to the variable $t$; the circular frequency $(p=2 \pi / T)(1.9)$

$\mathrm{p}_{\Gamma \mathrm{k}}$ - limiting frequency (the minimum possible) of the $\mathrm{k}$-th mode

$\mathrm{p}_{\mathrm{k}}^{2}-\mathrm{k}$-th characteristic value of the operator $(1.16-1.20)$

$\mathrm{p}$ - group-velocity frequency extremum (Airy phase) (1.64)

$\mathrm{R}_{0}$ - earth's radius

$\mathrm{r}$ - cylindrical coordinate; epicentral distance (1.11)

$\mathrm{r}_{\mathrm{k}}$ - auxiliary function used in the calculation $\mathrm{V}_{\mathrm{k}},(2.4)$

*The numbers in parentheses are those of the formulas in which the notation being described is used for the first time. 


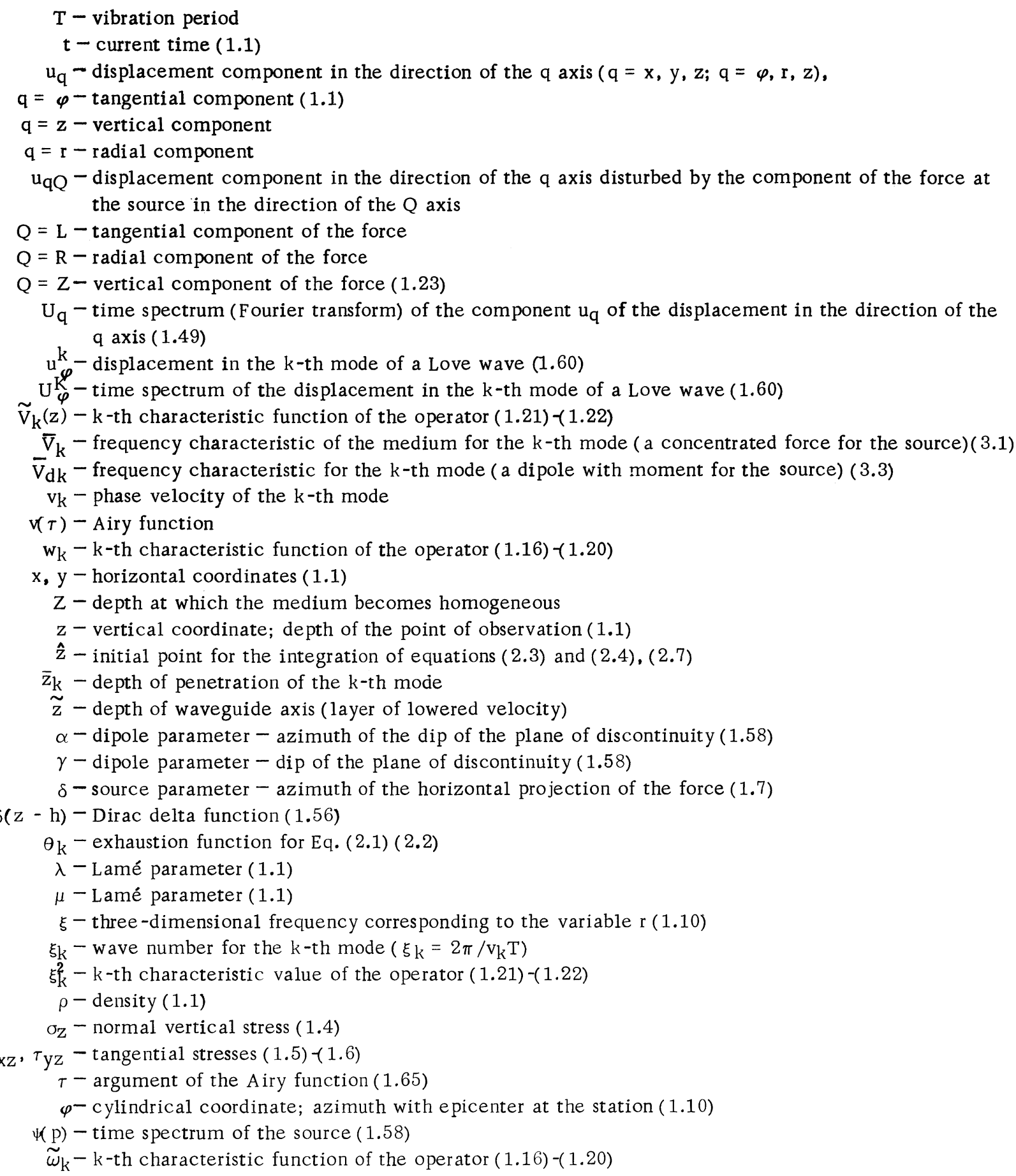

